\title{
Approximate Derivations with the Radical Ranges of Noncommutative Banach Algebras
}

\author{
Jaiok Roh ${ }^{1}$ and Ick-Soon Chang ${ }^{2}$ \\ ${ }^{1}$ Department of Mathematics, Hallym University, Chuncheon 200-702, Republic of Korea \\ ${ }^{2}$ Department of Mathematics, Chungnam National University, 79 Daehangno, Yuseong-gu, Daejeon 305-764, Republic of Korea \\ Correspondence should be addressed to Ick-Soon Chang; ischang@cnu.ac.kr
}

Received 24 June 2014; Accepted 22 July 2014

Academic Editor: Janusz Brzdęk

Copyright (c) 2015 J. Roh and I.-S. Chang. This is an open access article distributed under the Creative Commons Attribution License, which permits unrestricted use, distribution, and reproduction in any medium, provided the original work is properly cited.

We consider the derivations on noncommutative Banach algebras, and we will first study the conditions for a derivation on noncommutative Banach algebra. Then, we examine the stability of functional inequalities with a derivation. Finally, we take the derivations with the radical ranges on noncommutative Banach algebras.

\section{Introduction and Preliminaries}

Let $\mathscr{A}$ be an algebra over the real or complex field $\mathbb{F}$. An additive mapping $f: \mathscr{A} \rightarrow \mathscr{A}$ is called a ring derivation if the functional equation $f(x y)=x f(y)+f(x) y$ is valid for all $x, y \in \mathscr{A}$. In addition, if the identity $f(\lambda x)=\lambda f(x)$ holds for all $\lambda \in \mathbb{F}$ and all $x \in \mathscr{A}$, then $f$ is said to be a linear derivation.

Singer and Wermer [1] obtained a fundamental result which started investigation into the ranges of linear derivations on Banach algebras. The result states that every continuous linear derivation on a commutative Banach algebra maps into the radical. They also made a very insightful conjecture that the assumption of continuity is unnecessary. This conjecture was proved by Thomas [2]. So, in this paper, we will take into account the problems in $[1,2]$ for the derivations on noncommutative Banach algebras.

On the other hand, the stability problem for ring derivations on Banach algebras was considered by Miura et al. in [3]: under suitable conditions every approximate ring derivation on Banach algebra is an exact ring derivation. Šemrl [4] obtained the first stability result concerning derivations between operator algebras. As just mentioned, the study of stability problem has originally been formulated by Ulam [5]: under what condition does there exist a homomorphism near an approximate homomorphism? Hyers [6] had answered affirmatively the question of Ulam under the assumption that the groups are Banach spaces. A generalized version of the theorem of Hyers for approximately additive mappings was given by Aoki [7] and for approximately linear mappings was presented by Rassias [8] by considering an unbounded Cauchy difference. Since then, many interesting results of the stability problems to a number of functional equations and inequalities (or involving derivations) have been investigated (see, e.g., [9-16]). The reader is referred to the book [17] for more information on stability problem with a large variety of applications.

In this paper, we will establish the stability of functional inequalities with ring derivations and will deal with the problem for the radical range of these functional inequalities on Banach algebra by considering the base of noncommutative versions for the result of Singer and Wermer: Mathieu and Murphy [18] verify that every continuous centralizing linear derivation on a Banach algebra maps into the radical and Brešar [19] proved that every centralizing linear derivation on a semiprime Banach algebra maps into the intersection of the center and the radical. Moreover, Chaudhry and Thaheem [20] showed that two ring derivations on semiprime ring with suitable property map into the center. 


\section{Functional Inequalities for a Derivation and Its Applications}

Theorem 1. Let $\mathscr{A}$ be a normed algebra. Assume that mappings $\Phi: \mathscr{A}^{3} \rightarrow[0, \infty)$ and $\varphi: \mathscr{A}^{2} \rightarrow[0, \infty)$ satisfy the assumptions

$$
\begin{aligned}
& (1) \Phi(x / s, 0,-x / u)=\quad=\Phi(0, x / t,-x / u)= \\
& \Phi(-x / s, x / t, 0)=0,(x \in \mathscr{A}),
\end{aligned}
$$

(2) $\int \lim _{n \rightarrow \infty}(u / s)^{n} \Phi\left((s / u)^{n}(u x / s),(s / u)^{n}(u y / t)\right.$, $\left\{\begin{array}{l}\left.(s / u)^{n}(-x-y)\right)=0, \\ \lim _{n \rightarrow \infty}(u / s)^{2 n} \varphi\left((s / u)^{n} x,(s / u)^{n} y\right)=0, \\ (x, y \in \mathscr{A})\end{array}\right.$ or $\left\{\begin{array}{l}\lim _{n \rightarrow \infty}(s / u)^{n} \Phi\left((u / s)^{n+1} x,(u / s)^{n}(u y / t)\right. \\ \left.(u / s)^{n}(-x-y)\right)=0 \\ \lim _{n \rightarrow \infty}(s / u)^{2 n} \varphi\left((u / s)^{n} x,(u / s)^{n} y\right)=0 \\ (x, y \in \mathscr{A})\end{array}\right.$

where $s, t$, and $u$ are fixed positive real numbers with $s>u$ and $s+t+u>1$. Suppose that $f: \mathscr{A} \rightarrow \mathscr{A}$ is a mapping subjected to the inequalities

$$
\begin{aligned}
& \|s f(x)+t f(y)+u f(z)\| \\
& \quad \leq\|f(s x+t y+u z)\|+\Phi(x, y, z), \\
& \|f(x y)-x f(y)-f(x) y\| \\
& \quad \leq \varphi(x, y)
\end{aligned}
$$

for all $x, y, z \in \mathscr{A}$. Then $f$ is a ring derivation.

Proof. By letting $x=y=z=0$ in (1), we get $f(0)=0$. And by putting $x=x / s, y=0$, and $z=-x / u$ in (1), we obtain

$$
s f\left(\frac{x}{s}\right)=-u f\left(\frac{-x}{u}\right)
$$

for all $x \in \mathscr{A}$. Also, by letting $x=0, y=x / t$, and $z=-x / u$ in (1), it follows that

$$
t f\left(\frac{x}{t}\right)=-u f\left(\frac{-x}{u}\right)
$$

for all $x \in \mathscr{A}$. Replacing $x$ by $-x / s$ and setting $y=x / t$ and $z=0$ in (1), we have

$$
s f\left(\frac{-x}{s}\right)=-t f\left(\frac{x}{t}\right)
$$

for all $x \in \mathscr{A}$. From (3), (4), and (5), we arrive at

$$
f(-x)=-f(x)
$$

for all $x \in \mathscr{A}$. So the relations (3), (4), (5), and (6) give

$$
s f\left(\frac{x}{s}\right)=t f\left(\frac{x}{t}\right)=u f\left(\frac{x}{u}\right)
$$

for all $x \in \mathscr{A}$. Replacing $x$ by $x / s$ and letting $y=x / t$ and $z=z / u$ in (1), the following yields

$$
\begin{aligned}
& \left\|s f\left(\frac{x}{s}\right)+t f\left(\frac{y}{t}\right)+u f\left(\frac{z}{u}\right)\right\| \\
& \quad \leq\|f(x+y+z)\|+\Phi\left(\frac{x}{s}, \frac{y}{t}, \frac{z}{u}\right)
\end{aligned}
$$

for all $x, y, z \in \mathscr{A}$. Due to (7) and (8), we conclude that

$$
\begin{aligned}
& \|f(x)+f(y)+f(z)\| \\
& \quad \leq \frac{1}{u}\left[\|f(u(x+y+z))\|+\Phi\left(\frac{u x}{s}, \frac{u y}{t}, z\right)\right]
\end{aligned}
$$

for all $x, y, z \in \mathscr{A}$.

Next we are in the position to show that $f$ is a ring derivation. We will consider two different cases for the second assumption of $\Phi$.

Case I. Assume that

$$
\begin{aligned}
& \lim _{n \rightarrow \infty}\left(\frac{u}{s}\right)^{n} \Phi\left(\left(\frac{s}{u}\right)^{n} \frac{u x}{s},\left(\frac{s}{u}\right)^{n} \frac{u y}{t},\left(\frac{s}{u}\right)^{n}(-x-y)\right) \\
& \quad=0 \\
& \lim _{n \rightarrow \infty}\left(\frac{u}{s}\right)^{2 n} \varphi\left(\left(\frac{s}{u}\right)^{n} x,\left(\frac{s}{u}\right)^{n} y\right) \\
& \quad=0
\end{aligned}
$$

for all $x, y \in \mathscr{A}$. We get by (7)

$$
\begin{aligned}
f(x) & =\frac{u}{s} f\left(\frac{s}{u} x\right)=\left(\frac{u}{s}\right)^{2} f\left(\left(\frac{s}{u}\right)^{2} x\right)=\cdots \\
& =\left(\frac{u}{s}\right)^{n} f\left(\left(\frac{s}{u}\right)^{n} x\right)
\end{aligned}
$$

for all positive integers $n$ and all $x \in \mathscr{A}$. Therefore, one can obtain that

$$
f(x):=\lim _{n \rightarrow \infty}\left(\frac{u}{s}\right)^{n} f\left(\left(\frac{s}{u}\right)^{n} x\right)
$$

for all $x \in \mathscr{A}$. Due to (6) and (9), we see that

$$
\begin{aligned}
& \|f(x)+f(y)-f(x+y)\| \\
& =\lim _{n \rightarrow \infty}\left(\frac{u}{s}\right)^{n} \| f\left(\left(\frac{s}{u}\right)^{n} x\right)+f\left(\left(\frac{s}{u}\right)^{n} y\right) \\
& \quad+f\left(\left(\frac{s}{u}\right)^{n}(-x-y)\right) \| \\
& \leq \lim _{n \rightarrow \infty} \frac{1}{u}\left(\frac{u}{s}\right)^{n} \\
& \cdot \Phi\left(\left(\frac{s}{u}\right)^{n} \frac{u x}{s},\left(\frac{s}{u}\right)^{n} \frac{u y}{t},\left(\frac{s}{u}\right)^{n}(-x-y)\right)=0
\end{aligned}
$$

for all $x, y \in \mathscr{A}$. Thus $f(x+y)=f(x)+f(y)$. 
By (2), we find that

$$
\begin{aligned}
& \| f(x y)-x f(y)- f(x) y \| \\
&=\lim _{n \rightarrow \infty}\left(\frac{u}{s}\right)^{2 n} \| f\left(\left(\frac{s}{u}\right)^{2 n} x y\right) \\
&-\left(\frac{s}{u}\right)^{n} x f\left(\left(\frac{s}{u}\right)^{n} y\right) \\
&-\left(\frac{s}{u}\right)^{n} f\left(\left(\frac{s}{u}\right)^{n} x\right) y \| \\
& \leq \lim _{n \rightarrow \infty}\left(\frac{u}{s}\right)^{2 n} \varphi\left(\left(\frac{s}{u}\right)^{n} x,\left(\frac{s}{u}\right)^{n} y\right)=0
\end{aligned}
$$

for all $x, y \in \mathscr{A}$. Thus $f(x y)=x f(y)+f(x) y$.

Case II. Assume that

$$
\begin{aligned}
& \lim _{n \rightarrow \infty}\left(\frac{s}{u}\right)^{n} \Phi\left(\left(\frac{u}{s}\right)^{n+1} x,\left(\frac{u}{s}\right)^{n} \frac{u y}{t},\left(\frac{u}{s}\right)^{n}(-x-y)\right) \\
& =0 \\
& \lim _{n \rightarrow \infty}\left(\frac{s}{u}\right)^{2 n} \varphi\left(\left(\frac{u}{s}\right)^{n} x,\left(\frac{u}{s}\right)^{n} y\right)=0
\end{aligned}
$$

for all $x, y \in \mathscr{A}$. We get by (7)

$$
\begin{aligned}
f(x) & =\frac{s}{u} f\left(\frac{u}{s} x\right)=\left(\frac{s}{u}\right)^{2} f\left(\left(\frac{u}{s}\right)^{2} x\right)=\cdots \\
& =\left(\frac{s}{u}\right)^{n} f\left(\left(\frac{u}{s}\right)^{n} x\right)
\end{aligned}
$$

for all positive integers $n$ and all $x \in \mathscr{A}$. Therefore, one can obtain that

$$
f(x):=\lim _{n \rightarrow \infty}\left(\frac{s}{u}\right)^{n} f\left(\left(\frac{u}{s}\right)^{n} x\right)
$$

for all $x \in \mathscr{A}$. The remainder of the proof is similar to the proof of Case I.

Corollary 2. Let $\mathscr{A}$ be a Banach algebra and let $s, t$, and $u$ be fixed positive real numbers with $s>u$ and $s+t+u>1$. Suppose that mapping $\Phi: \mathscr{A}^{3} \rightarrow[0, \infty)$ satisfies the assumption (1) and the first case of assumption (2) in Theorem 1. Assume that $f: \mathscr{A} \rightarrow \mathscr{A}$ is a continuous mapping subjected to the inequality

$$
\begin{aligned}
& \|\alpha s f(x)+t f(y)+u f(\alpha z)\| \\
& \quad \leq\|f(s x+t y+u z)\|+\Phi(x, y, z)
\end{aligned}
$$

for all $x, y, z \in \mathscr{A}$ and all $\alpha=1, i$, where $i \in \mathbb{C}$. In addition, if a mapping $f$ is fulfilled with the following inequalities:

$$
\begin{array}{r}
\|f(x y)-x f(y)-f(x) y\| \leq \varepsilon, \\
\|[[f(x), x], y]\| \leq \delta
\end{array}
$$

for some $\varepsilon>0$ and $\delta>0$ and all $x, y \in \mathscr{A}$, where $[x, y]=$ $x y-y x$, then $f$ maps $\mathscr{A}$ into its radical $\operatorname{rad}(\mathscr{A})$.
Proof. We first consider $\alpha=1$ in (18) and $\varepsilon:=\varphi(x, y)$ in (19). It follows from Theorem 1 that $f$ is a ring derivation. In this case, $f$ is a mapping defined by $f(x)$ := $\lim _{n \rightarrow \infty}(u / s)^{n} f\left((s / u)^{n} x\right)$ for all $x \in \mathscr{A}$ and $f$ satisfies (7). Since $f$ is continuous, $f(t x)$ is continuous in $t \in \mathbb{R}$ for each fixed $x \in \mathscr{A}$. Thus $f$ is $\mathbb{R}$-linear as in [8]. Replacing $x$ by $x / s$ and putting $\alpha=i, y=0$, and $z=-x / u$ in (18) with (7), we see that $f(i x)=i f(x)$ and so we see that $f(\mu x)=s f(x)+i t f(x)=$ $\mu f(x)$ for all $x \in \mathscr{A}$ and all $\mu=s+i t \in \mathbb{C}$. Hence $f$ is $\mathbb{C}$-linear.

In view of (20), we have

$$
\begin{aligned}
& \lim _{n \rightarrow \infty}\left(\frac{u}{s}\right)^{n}\left\|\left[\left[f\left(\left(\frac{s}{u}\right)^{n} x\right),\left(\frac{s}{u}\right)^{n} x\right], y\right]\right\| \\
& \leq \lim _{n \rightarrow \infty}\left(\frac{u}{s}\right)^{n} \delta=0
\end{aligned}
$$

for all $x, y \in \mathscr{A}$, which means that $f$ is a centralizing mapping. With the help of Mathieu and Murphy's result [18], we arrive at the conclusion.

Corollary 3. Let $\mathscr{A}$ be a Banach algebra with identity and let $s, t$, and $u$ be fixed positive real numbers with $s>u$ and $s+t+u>1$. Suppose that mapping $\Phi: \mathscr{A}^{3} \rightarrow[0, \infty)$ satisfies the assumption (1) and the second case of assumption (2) in Theorem 1 and suppose that $f: \mathscr{A} \rightarrow \mathscr{A}$ is a continuous centralizing mapping subjected to (18) and (19). Then $f$ maps $\mathscr{A}$ into its radical $\mathrm{rad}(\mathscr{A})$.

Proof. We let $\alpha=1$ in (18). In the proof of Theorem 1, we find that $f$ is an additive mapping. In this case, $f$ is a mapping defined by $f(x):=\lim _{n \rightarrow \infty}(s / u)^{n} f\left((u / s)^{n} x\right)$ for all $x \in \mathscr{A}$ and $f$ satisfies (7). As in the proof of the Corollary 2, the mapping $f$ is linear. Since $\mathscr{A}$ contains the identity, Badora's result [10] implies that $f(x y)=x f(y)+f(x) y$ for all $x, y \in$ $\mathscr{A}$. So $f$ is a centralizing linear derivation. Based on the result of Mathieu and Murphy [18], we conclude that $f(\mathscr{A}) \subseteq$ $\operatorname{rad}(\mathscr{A})$.

\section{Approximate Derivations and Their Applications}

Theorem 4. Let $\mathscr{A}$ be a Banach algebra. Assume that mappings $\Phi: \mathscr{A}^{3} \rightarrow[0, \infty)$ and $\varphi: \mathscr{A}^{2} \rightarrow[0, \infty)$ satisfy the following assumptions:

(1) $\sum_{j=0}^{\infty}(u / s)^{j}\left[(2 / s) \Phi\left((s / u)^{j} x, 0,-(s / u)^{j+1} x\right)+(2 / s) \Phi(0\right.$, $\left.-(s / u)^{j+1}(u x / t),(s / u)^{j+1} x\right)+(1 / s) \Phi\left((s / u)^{j} x\right.$, $\left.\left.-(s / u)^{j+1}(u x / t), 0\right)\right]<\infty,(x \in \mathscr{A})$,

(2) $\lim _{n \rightarrow \infty}(u / s)^{n} \Phi\left((s / u)^{n} x,(s / u)^{n} y,(s / u)^{n} z\right)=0$, $(x, y, z \in \mathscr{A}), \lim _{n \rightarrow \infty}(u / s)^{n} \varphi\left((s / u)^{n} x, y\right)=0,(x, y \in$ $\mathscr{A})$

where $s, t$, and $u$ are fixed positive real numbers with $s>u$ and $s+t+u>1$. Suppose that $f: \mathscr{A} \rightarrow \mathscr{A}$ is a mapping subjected to the inequalities (1) and (2). Then, there exists a unique ring derivation $\mathscr{L}: \mathscr{A} \rightarrow \mathscr{A}$ such that the inequality

$$
\|\mathscr{L}(x)-f(x)\| \leq \eta(x)
$$


for all $x \in \mathscr{A}$, where

$$
\begin{aligned}
\eta(x)=\sum_{j=0}^{\infty}\left(\frac{u}{s}\right)^{j}[ & \frac{2}{s} \Phi\left(\left(\frac{s}{u}\right)^{j} x, 0,-\left(\frac{s}{u}\right)^{j+1} x\right) \\
& +\frac{2}{s} \Phi\left(0,-\left(\frac{s}{u}\right)^{j+1} \frac{u x}{t},\left(\frac{s}{u}\right)^{j+1} x\right) \\
& +\frac{1}{s} \Phi\left(\left(\frac{s}{u}\right)^{j} x,-\left(\frac{s}{u}\right)^{j+1} \frac{u x}{t}, 0\right) \\
& \left.+\frac{s+2 t+u+3}{s(s+t+u-1)} \Phi(0,0,0)\right] .
\end{aligned}
$$

In addition, the equation

$$
x\{f(y)-\mathscr{L}(y)\}=0
$$

holds for all $x \in \mathscr{A}$.

Proof. By letting $x=y=z=0$ in (1), we get $\|f(0)\| \leq$ $(1 /(s+t+u-1)) \Phi(0,0,0)$. Replacing $x$ by $x=x / s$ and letting $y=0$ and $z=-x / u$ in (1), we also have

$$
\begin{aligned}
& \left\|s f\left(\frac{x}{s}\right)+u f\left(\frac{-x}{u}\right)\right\| \\
& \quad \leq \Phi\left(\frac{x}{s}, 0, \frac{-x}{u}\right)+\frac{t+1}{s+t+u-1} \Phi(0,0,0)
\end{aligned}
$$

for all $x \in \mathscr{A}$, which implies that

$$
\begin{aligned}
\| f & (x)+\frac{u}{s} f\left(\frac{-s x}{u}\right) \| \\
& \leq \frac{1}{s}\left[\Phi\left(x, 0, \frac{-s x}{u}\right)+\frac{t+1}{s+t+u-1} \Phi(0,0,0)\right] .
\end{aligned}
$$

Next, by letting $x=0, y=x / t$, and $z=-x / u$ in (1), we obtain

$$
\begin{aligned}
& \left\|t f\left(\frac{x}{t}\right)+u f\left(\frac{-x}{u}\right)\right\| \\
& \quad \leq \Phi\left(0, \frac{x}{t}, \frac{-x}{u}\right)+\frac{s+1}{s+t+u-1} \Phi(0,0,0)
\end{aligned}
$$

for all $x \in \mathscr{A}$. Again, replacing $x$ by $-x / s$ and setting $y=x / t$ and $z=0$ in (1), we find that

$$
\begin{aligned}
\| s f & \left(\frac{-x}{s}\right)+t f\left(\frac{x}{t}\right) \| \\
& \leq \Phi\left(\frac{-x}{s}, \frac{x}{t}, 0\right)+\frac{u+1}{s+t+u-1} \Phi(0,0,0)
\end{aligned}
$$

for all $x \in \mathscr{A}$. It follows from (25), (27), and (28) that

$$
\begin{aligned}
\left\|u f\left(\frac{x}{u}\right)+u f\left(\frac{-x}{u}\right)\right\| \\
\leq\left\|s f\left(\frac{x}{s}\right)+u f\left(\frac{-x}{u}\right)\right\|+\left\|u f\left(\frac{x}{u}\right)+t f\left(\frac{-x}{t}\right)\right\| \\
\quad+\left\|s f\left(\frac{x}{s}\right)+t f\left(\frac{-x}{t}\right)\right\| \\
\leq \Phi\left(\frac{x}{s}, 0, \frac{-x}{u}\right)+\Phi\left(0, \frac{-x}{t}, \frac{x}{u}\right)+\Phi\left(\frac{x}{s}, \frac{-x}{t}, 0\right) \\
\quad+\frac{s+t+u+3}{s+t+u-1} \Phi(0,0,0)
\end{aligned}
$$

for all $x \in \mathscr{A}$. So the relation (29) can be rewritten as

$$
\begin{aligned}
& \|f(x)+f(-x)\| \\
& \leq \frac{1}{u}\left[\Phi\left(\frac{u x}{s}, 0,-x\right)+\Phi\left(0, \frac{-u x}{t}, x\right)\right. \\
& \left.\quad+\Phi\left(\frac{u x}{s}, \frac{-u x}{t}, 0\right)+\frac{s+t+u+3}{s+t+u-1} \Phi(0,0,0)\right]
\end{aligned}
$$

for all $x \in \mathscr{A}$. Therefore, by (26) and (30), we see that

$$
\begin{gathered}
\left\|\left(\frac{u}{s}\right)^{l} f\left(\left(\frac{s}{u}\right)^{l} x\right)-\left(\frac{u}{s}\right)^{m} f\left(\left(\frac{s}{u}\right)^{m} x\right)\right\| \\
\leq \sum_{j=l}^{m-1}\left\|\left(\frac{u}{s}\right)^{j} f\left(\left(\frac{s}{u}\right)^{j} x\right)-\left(\frac{u}{s}\right)^{j+1} f\left(\left(\frac{s}{u}\right)^{j+1} x\right)\right\| \\
\leq \sum_{j=l}^{m-1}\left(\frac{u}{s}\right)^{j}\left[\frac{2}{s} \Phi\left(\left(\frac{s}{u}\right)^{j} x, 0,-\left(\frac{s}{u}\right)^{j+1} x\right)\right. \\
+\frac{2}{s} \Phi\left(0,-\left(\frac{s}{u}\right)^{j+1} \frac{u x}{t},\left(\frac{s}{u}\right)^{j+1} x\right) \\
+\frac{1}{s} \Phi\left(\left(\frac{s}{u}\right)^{j} x,-\left(\frac{s}{u}\right)^{j+1} \frac{u x}{t}, 0\right) \\
\left.+\frac{s+2 t+u+3}{s(s+t+u-1)} \Phi(0,0,0)\right]
\end{gathered}
$$

for all nonnegative integers $m, l$ with $m>l$ and all $x \in \mathscr{A}$. This means that $\left\{(u / s)^{n} f\left((s / u)^{n} x\right)\right\}$ is a Cauchy sequence. Hence the sequence $\left\{(u / s)^{n} f\left((s / u)^{n} x\right)\right\}$ converges. So one can define a mapping $\mathscr{L}: \mathscr{A} \rightarrow \mathscr{A}$ by $\mathscr{L}(x)$ := $\lim _{n \rightarrow \infty}(u / s)^{n} f\left((s / u)^{n} x\right)$ for all $x \in \mathscr{A}$. Letting $l=0$ and taking the limit $m \rightarrow \infty$, we arrive at (22).

Now we claim that the mapping $\mathscr{L}$ is additive. By (30), one notes

$$
\begin{aligned}
& \|\mathscr{L}(x)+\mathscr{L}(-x)\| \\
& =\lim _{n \rightarrow \infty}\left(\frac{u}{s}\right)^{n}\left\|f\left(\left(\frac{s}{u}\right)^{n} x\right)+f\left(-\left(\frac{s}{u}\right)^{n} x\right)\right\| \\
& \leq \lim _{n \rightarrow \infty} \frac{1}{u}\left(\frac{u}{s}\right)^{n} \\
& \quad \cdot\left[\Phi\left(\left(\frac{s}{u}\right)^{n} \frac{u x}{s}, 0,-\left(\frac{s}{u}\right)^{n} x\right)\right. \\
& \quad+\Phi\left(0,-\left(\frac{s}{u}\right)^{n} \frac{u x}{t},\left(\frac{s}{u}\right)^{n} x\right) \\
& \quad+\Phi\left(\left(\frac{s}{u}\right)^{n} \frac{u x}{s},-\left(\frac{s}{u}\right)^{n} \frac{u x}{t}, 0\right) \\
& \left.+\frac{s+t+u+3}{s+t+u-1} \Phi(0,0,0)\right]
\end{aligned}
$$


for all $x \in \mathscr{A}$. So we have $\mathscr{L}(-x)=-\mathscr{L}(x)$. By virtue of $(25)$, we get

$$
\begin{aligned}
& \left\|s \mathscr{L}\left(\frac{x}{s}\right)+u \mathscr{L}\left(\frac{-x}{u}\right)\right\| \\
& =\lim _{n \rightarrow \infty}\left(\frac{u}{s}\right)^{n}\left\|s f\left(\left(\frac{s}{u}\right)^{n} \frac{x}{s}\right)+u f\left(-\left(\frac{s}{u}\right)^{n} \frac{x}{u}\right)\right\| \\
& \leq \lim _{n \rightarrow \infty}\left(\frac{u}{s}\right)^{n}\left[\Phi\left(\left(\frac{s}{u}\right)^{n} \frac{x}{s}, 0,-\left(\frac{s}{u}\right)^{n} \frac{x}{u}\right)\right. \\
& \left.+\frac{t+1}{s+t+u-1} \Phi(0,0,0)\right]=0
\end{aligned}
$$

for all $x \in \mathscr{A}$, which means that $s \mathscr{L}(x / s)=u \mathscr{L}(x / u)$. Using the similar way with (27), we feel that $t \mathscr{L}(x / t)=u \mathscr{L}(x / u)$. Therefore, we see that

$$
s \mathscr{L}\left(\frac{x}{s}\right)=t \mathscr{L}\left(\frac{x}{t}\right)=u \mathscr{L}\left(\frac{x}{u}\right),
$$

which yields that

$$
\begin{aligned}
\mathscr{L}(x) & =\frac{u}{s} \mathscr{L}\left(\frac{s}{u} x\right)=\left(\frac{u}{s}\right)^{2} \mathscr{L}\left(\left(\frac{s}{u}\right)^{2} x\right)=\cdots \\
& =\left(\frac{u}{s}\right)^{n} \mathscr{L}\left(\left(\frac{s}{u}\right)^{n} x\right)
\end{aligned}
$$

for all $x \in \mathscr{A}$. By (1), we obtain

$$
\begin{aligned}
& \left\|s \mathscr{L}\left(\frac{x}{s}\right)+t \mathscr{L}\left(\frac{y}{t}\right)-u \mathscr{L}\left(\frac{x+y}{u}\right)\right\| \\
& =\lim _{n \rightarrow \infty}\left(\frac{u}{s}\right)^{n} \\
& \quad \cdot\left\|s f\left(\left(\frac{s}{u}\right)^{n} \frac{x}{s}\right)+t f\left(\left(\frac{s}{u}\right)^{n} \frac{y}{t}\right)+u f\left(-\left(\frac{s}{u}\right)^{n} \frac{x+y}{u}\right)\right\| \\
& \leq \lim _{n \rightarrow \infty}\left(\frac{u}{s}\right)^{n} \\
& \quad \cdot\left[\Phi\left(\left(\frac{s}{u}\right)^{n} \frac{x}{s},\left(\frac{s}{u}\right)^{n} \frac{y}{s},-\left(\frac{s}{u}\right)^{n} \frac{x+y}{u}\right)\right. \\
& \left.\quad+\frac{1}{s+t+u-1} \Phi(0,0,0)\right]=0
\end{aligned}
$$

for all $x, y \in \mathscr{A}$. So we know that

$$
u \mathscr{L}\left(\frac{x+y}{u}\right)=s \mathscr{L}\left(\frac{x}{s}\right)+t \mathscr{L}\left(\frac{y}{t}\right) .
$$

Due to (34) and (37), we conclude that $\mathscr{L}(x+y)=\mathscr{L}(x)+$ $\mathscr{L}(y)$.

In particular, by (2), we note that

$$
\begin{aligned}
& \|\mathscr{L}(x y)-x f(y)-\mathscr{L}(x) y\| \\
& =\lim _{n \rightarrow \infty}\left(\frac{u}{s}\right)^{n} \\
& \quad \cdot\left\|f\left(\left(\frac{s}{u}\right)^{n} x y\right)-\left(\frac{s}{u}\right)^{n} x f(y)-f\left(\left(\frac{s}{u}\right)^{n} x\right) y\right\| \\
& \leq \lim _{n \rightarrow \infty}\left(\frac{u}{s}\right)^{n} \varphi\left(\left(\frac{s}{u}\right)^{n} x, y\right)=0
\end{aligned}
$$

for all $x, y \in \mathscr{A}$. Thus we get

$$
\mathscr{L}(x y)=x f(y)+\mathscr{L}(x) y .
$$

The conditions (35) and (39) guarantee that

$$
\begin{aligned}
& \left(\frac{s}{u}\right)^{n} x f(y)+\left(\frac{s}{u}\right)^{n} \mathscr{L}(x) y \\
& =\mathscr{L}\left(\left(\frac{s}{u}\right)^{n} x \cdot y\right)=\mathscr{L}\left(x \cdot\left(\frac{s}{u}\right)^{n} y\right) \\
& =x f\left(\left(\frac{s}{u}\right)^{n} y\right)+\left(\frac{s}{u}\right)^{n} \mathscr{L}(x) y,
\end{aligned}
$$

which implies that

$$
x f(y)=\lim _{n \rightarrow \infty}\left(\frac{u}{s}\right)^{n} x f\left(\left(\frac{s}{u}\right)^{n} y\right)=x \mathscr{L}(y) .
$$

Therefore, we obtain $(24)$ and $\mathscr{L}(x y)=x \mathscr{L}(y)+\mathscr{L}(x) y$.

Now, to show uniqueness of the mapping $\mathscr{L}$, let us assume that $T: \mathscr{A} \rightarrow \mathscr{A}$ is another ring derivation satisfying (22). Then, we have by (22) and (35)

$$
\begin{aligned}
& \|\mathscr{L}(x)-T(x)\| \\
& =\lim _{n \rightarrow \infty}\left(\frac{u}{s}\right)^{n}\left\|\mathscr{L}\left(\left(\frac{s}{u}\right)^{n} x\right)-T\left(\left(\frac{s}{u}\right)^{n} x\right)\right\| \\
& \leq \lim _{n \rightarrow \infty}\left(\frac{u}{s}\right)^{n} \\
& \quad \cdot\left[\left\|\mathscr{L}\left(\left(\frac{s}{u}\right)^{n} x\right)-f\left(\left(\frac{s}{u}\right)^{n} x\right)\right\|\right. \\
& \left.\quad+\left\|f\left(\left(\frac{s}{u}\right)^{n} x\right)-\mathscr{L}\left(\left(\frac{s}{u}\right)^{n} x\right)\right\|\right] \\
& \leq \lim _{n \rightarrow \infty} 2\left(\frac{u}{s}\right)^{n} \eta\left(\left(\frac{s}{u}\right)^{n} x\right)=0
\end{aligned}
$$

for all $x \in \mathscr{A}$, which means that $\mathscr{L}=T$.

From now on, we suppose that $\mathbb{U}:=\{z \in \mathbb{C}:|z|=1\}$.

Corollary 5. Let $\mathscr{A}$ be a semiprime Banach algebra. Assume that the mappings $\Phi: \mathscr{A}^{3} \rightarrow[0, \infty)$ and $\varphi: \mathscr{A}^{2} \rightarrow[0, \infty)$ satisfy the assumptions of Theorem 4. Suppose that $f: \mathscr{A} \rightarrow$ $\mathscr{A}$ is a mapping such that the inequality (18) holds for all $x, y, z \in \mathscr{A}$ and all $\alpha \in \mathbb{U}$. Moreover, if a mapping $f$ satisfies the conditions (2) and (20), then $f$ maps $\mathscr{A}$ into the intersection of its center $Z(\mathscr{A})$ and its radical $\mathrm{rad}(\mathscr{A})$.

Proof. Now we take $\alpha=1$ in (18). Replacing $(f(y)-\mathscr{L}(y)) x$ instead of $x$ in (24), we see that $(f(y)-\mathscr{L}(y)) x(f(y)-$ $\mathscr{L}(y))=0$ for all $x, y \in \mathscr{A}$. Since $\mathscr{A}$ is semiprime, we conclude that $f=\mathscr{L}$. So Theorem 4 provides that $f$ is a ring derivation and one can obtain that $f(x):=\lim _{n \rightarrow \infty}$ $(u / s)^{n} f\left((s / u)^{n} x\right)$ for all $x \in \mathscr{A}$. Moreover, $s f(x / s)=u f(x / u)$. Replacing $x$ by $x / s$ and setting $y=0$ and $z=-x / u$ in (18), then we get

$$
\begin{aligned}
& \left\|\alpha s f\left(\frac{x}{s}\right)+u f\left(\frac{-\alpha x}{u}\right)\right\| \\
& \quad \leq \Phi\left(\frac{x}{s}, 0, \frac{-x}{u}\right)+\frac{t+1}{s+t+u-1} \Phi(0,0,0) .
\end{aligned}
$$


This implies that

$$
\begin{gathered}
\lim _{n \rightarrow \infty}\left(\frac{u}{s}\right)^{n}\left\|\alpha s f\left(\left(\frac{s}{u}\right)^{n} \frac{x}{s}\right)+u f\left(\alpha\left(\frac{s}{u}\right)^{n} \frac{-x}{u}\right)\right\| \\
\leq \lim _{n \rightarrow \infty}\left(\frac{u}{s}\right)^{n}\left[\Phi\left(\left(\frac{s}{u}\right)^{n} \frac{x}{s}, 0,\left(\frac{s}{u}\right)^{n} \frac{-x}{u}\right)\right. \\
\left.+\frac{t+1}{s+t+u-1} \Phi(0,0,0)\right]=0 .
\end{gathered}
$$

Thus $f(\alpha x)=\alpha f(x)$ for all $x \in \mathscr{A}$ and all $\alpha \in \mathbb{U}$. Clearly, $f(0 x)=0 f(x)$. In addition, we prove that $f(\lambda x)=\lambda f(x)$ holds for all $x \in \mathscr{A}$ and all $\lambda \in \mathbb{C}$ (see [21]). So $f$ is linear. As in the proof of Corollary 2, we find that $f$ is a centralizing linear derivation. Therefore Brešar's result [19] yields our claim.

Theorem 6. Let $\mathscr{A}$ be a Banach algebra. Assume that mappings $\Phi: \mathscr{A}^{3} \rightarrow[0, \infty)$ and $\varphi: \mathscr{A}^{2} \rightarrow[0, \infty)$ satisfy the assumptions

(1) $\rho(x)=\sum_{j=0}^{\infty}(1 / u)(s / u)^{j}\left[\Phi\left(-(u / s)^{j+1} x, 0,(u / s)^{j} x\right)\right.$ $+(s / u)\left(\Phi\left((u / s)^{j+2} x, 0,-(u / s)^{j+1} x\right)+\Phi(0\right.$, $\left.-(u / s)^{j+1}(u x / t),(u / s)^{j+1} x\right)+\Phi\left((u / s)^{j+2} x\right.$, $\left.\left.\left.-(u / s)^{j+1}(u x / t), 0\right)\right)\right]<\infty,(x \in \mathscr{A})$,

(2) $\lim _{n \rightarrow \infty}(s / u)^{n} \Phi\left((u / s)^{n}(x),(u / s)^{n} y,(u / s)^{n} z\right)=0$, $(x, y \in \mathscr{A}), \lim _{n \rightarrow \infty}(s / u)^{2 n} \varphi\left((u / s)^{n} x, y\right)=$ $0,(x, y \in \mathscr{A})$,

where $s, t$, and $u$ are fixed positive real numbers with $s>u$ and $s+t+u>1$. Suppose that $f: \mathscr{A} \rightarrow \mathscr{A}$ is a mapping subjected to inequalities (1) and (2). Then there exists a unique ring derivation $\mathscr{L}: \mathscr{A} \rightarrow \mathscr{A}$ such that the inequality

$$
\|\mathscr{L}(x)-f(x)\| \leq \rho(x)
$$

for all $x \in \mathscr{A}$. Moreover, (24) holds.

Proof. Letting $x=y=z=0$ in (1), we get $\|f(0)\| \leq$ $(1 /(s+t+u-1)) \Phi(0,0,0)$. By assumption of $\Phi$, we should have $\Phi(0,0,0)=0$. Thus $f(0)=0$. Replacing $x$ by $-u x$ in (25) and then dividing $u$ in the resulting inequality,

$$
\left\|f(x)+\frac{s}{u} f\left(\frac{-u x}{s}\right)\right\| \leq \frac{1}{u} \Phi\left(\frac{-u x}{s}, 0, x\right)
$$

for all $x \in \mathscr{A}$. Starting from (25) and employing the same argument in the proof of the previous theorem, we obtain

$$
\begin{aligned}
& \|f(x)+f(-x)\| \\
& \leq \frac{1}{u}\left[\Phi\left(\frac{u x}{s}, 0,-x\right)+\Phi\left(0, \frac{-u x}{t}, x\right)+\Phi\left(\frac{u x}{s}, \frac{-u x}{t}, 0\right)\right]
\end{aligned}
$$

for all $x \in \mathscr{A}$. The rest of proof can be carried out similarly as the corresponding part of Theorem 4 .

Corollary 7. Let $\mathscr{A}$ be a semiprime Banach algebra. Assume that a mapping $\Phi: \mathscr{A}^{3} \rightarrow[0, \infty)$ satisfies the assumptions of Theorem 6 and that $f: \mathscr{A} \rightarrow \mathscr{A}$ is a centralizing mapping such that the inequality (18) holds for all $x, y, z \in \mathscr{A}$ and all $\alpha \in \mathbb{U}$. Suppose that a mapping $f$ is fulfilled with the inequality (19). Then $f$ maps $\mathscr{A}$ into the intersection of its center $Z(\mathscr{A})$ and its radical rad $(\mathscr{A})$.
Proof. Employing the same method in the proof of Corollary 5, we find that $f$ is linear derivation. According to Brešar's result [19], we get the result.

Corollary 8. Let $\mathscr{A}$ be a semiprime Banach algebra with identity. Suppose that mappings $\Phi: \mathscr{A}^{2} \rightarrow[0, \infty)$ and $\varphi:$ $\mathscr{A}^{2} \rightarrow[0, \infty)$ fulfilled the assumptions of Theorem 4 (resp., Theorem 6). Assume that $f_{1}: \mathscr{A} \rightarrow \mathscr{A}$ and $f_{2}: \mathscr{A} \rightarrow \mathscr{A}$ are mappings such that, for each $k=0,1$,

$$
\begin{aligned}
& \left\|\alpha s f_{k}(x)+t f_{k}(y)+u f_{k}(\alpha z)\right\| \\
& \quad \leq\left\|f_{k}(s x+t y+u z)\right\|+\Phi(x, y, z) \\
& \left\|f_{k}(x y)-x f_{k}(y)-f_{k}(x) y\right\| \\
& \quad \leq \varphi(x, y)
\end{aligned}
$$

for all $x, y, z \in \mathscr{A}$ and all $\alpha \in \mathbb{U}$. If $f_{1}(x) x+x f_{2}(x) \in Z(\mathscr{A})$ for all $x \in \mathscr{A}$, then $f_{1}$ and $f_{2}$ map $\mathscr{A}$ into the intersection of its center $Z(\mathscr{A})$ and its radical $\operatorname{rad}(\mathscr{A})$.

Proof. Employing the same argument in the proof of the previous corollaries, we see that $f_{1}$ and $f_{2}$ are linear derivations. Chaudhry and Thaheem's result [20] guarantees that $f_{1}(\mathscr{A}) \subseteq$ $Z(\mathscr{A})$ and $f_{2}(\mathscr{A}) \subseteq Z(\mathscr{A})$. This implies that $f_{1}$ and $f_{2}$ are centralizing mappings. Hence, by Brešar's result [19], we see that $f_{1}$ and $f_{2}$ map $\mathscr{A}$ into $Z(\mathscr{A}) \cap \operatorname{rad}(\mathscr{A})$.

\section{Conflict of Interests}

The authors declare that they have no conflict of interests regarding the publication of this paper.

\section{Acknowledgments}

The authors would like to thank the referees for giving useful suggestions and for the improvement of this paper. This research was supported by the Basic Science Research Program through the National Research Foundation of Korea (NRF) funded by the Ministry of Education (no. 2010002338 and no. 2013R1A1A2A10004419). This research was also supported by the National Research Foundation of Korea (NRF) funded by the Ministry of Science, ICT and Future Planning (no. 2012R1A1A2021721).

\section{References}

[1] I. M. Singer and J. Wermer, "Derivations on commutative normed algebras," Mathematische Annalen, vol. 129, pp. 260264, 1955.

[2] M. P. Thomas, "The image of a derivation is contained in the radical," Annals of Mathematics. Second Series, vol. 128, no. 3, pp. 435-460, 1988.

[3] T. Miura, G. Hirasawa, and S.-E. Takahasi, "A perturbation of ring derivations on Banach algebras," Journal of Mathematical Analysis and Applications, vol. 319, no. 2, pp. 522-530, 2006.

[4] P. Šemrl, "The functional equation of multiplicative derivation is superstable on standard operator algebras," Integral Equations and Operator Theory, vol. 18, no. 1, pp. 118-122, 1994. 
[5] S. M. Ulam, A Collection of Mathematical Problems, Interscience, New York, NY, USA, 1960.

[6] D. H. Hyers, "On the stability of the linear functional equation," Proceedings of the National Academy of Sciences of the United States of America, vol. 27, pp. 222-224, 1941.

[7] T. Aoki, "On the stability of the linear transformation in Banach spaces," Journal of the Mathematical Society of Japan, vol. 2, pp. 64-66, 1950.

[8] T. M. Rassias, "On the stability of the linear mapping in Banach spaces," Proceedings of the American Mathematical Society, vol. 72, no. 2, pp. 297-300, 1978.

[9] R. Badora, "On approximate ring homomorphisms," Journal of Mathematical Analysis and Applications, vol. 276, no. 2, pp. 589597, 2002.

[10] R. Badora, "On approximate derivations," Mathematical Inequalities \& Applications, vol. 9, no. 1, pp. 167-173, 2006.

[11] D. G. Bourgin, "Approximately isometric and multiplicative transformations on continuous function rings," Duke Mathematical Journal, vol. 16, pp. 385-397, 1949.

[12] J. Brzdęk and K. Ciepliński, "Hyperstability and superstability," Abstract and Applied Analysis, vol. 2013, Article ID 401756, 13 pages, 2013.

[13] I.-S. Chang, "Higher ring derivation and intuitionistic fuzzy stability," Abstract and Applied Analysis, vol. 2012, Article ID 503671, 16 pages, 2012.

[14] B. Hegyi and S.-M. Jung, "On the stability of heat equation," Abstract and Applied Analysis, vol. 2013, Article ID 202373, 4 pages, 2013.

[15] S. M. Jung, M. S. Moslehian, and P. K. Sahoo, "Stability of a generalized Jensen equation on restricted domains," Journal of Mathematical Inequalities, vol. 4, no. 2, pp. 191-205, 2010.

[16] F. Polat, "Approximate riesz algebra-valued derivations," Abstract and Applied Analysis, vol. 2012, Article ID 240258, 5 pages, 2012.

[17] S.-M. Jung, Hyers-Ulam-Rassias Stability of Functional Equations in Nonlinear Analysis, vol. 48 of Springer Optimization and Its Applications, Springer, New York, NY, USA, 2011.

[18] M. Mathieu and G. J. Murphy, "Derivations mapping into the radical," Archiv der Mathematik, vol. 57, no. 5, pp. 469-474, 1991.

[19] M. Brešar, "Centralizing mappings on von Neumann algebras," Proceedings of the American Mathematical Society, vol. 111, no. 2, pp. 501-510, 1991.

[20] M. A. Chaudhry and A. B. Thaheem, "A note on a pair of derivations of semiprime rings," International Journal of Mathematics and Mathematical Sciences, vol. 2004, no. 39, pp. 2097-2102, 2004.

[21] H.-M. Kim and I.-S. Chang, "Approximate linear derivations and functional inequalities with applications," Applied Mathematics Letters, vol. 25, no. 5, pp. 830-836, 2012. 


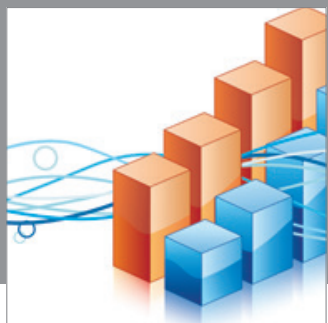

Advances in

Operations Research

mansans

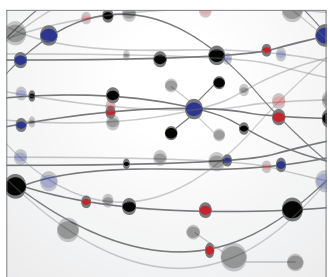

The Scientific World Journal
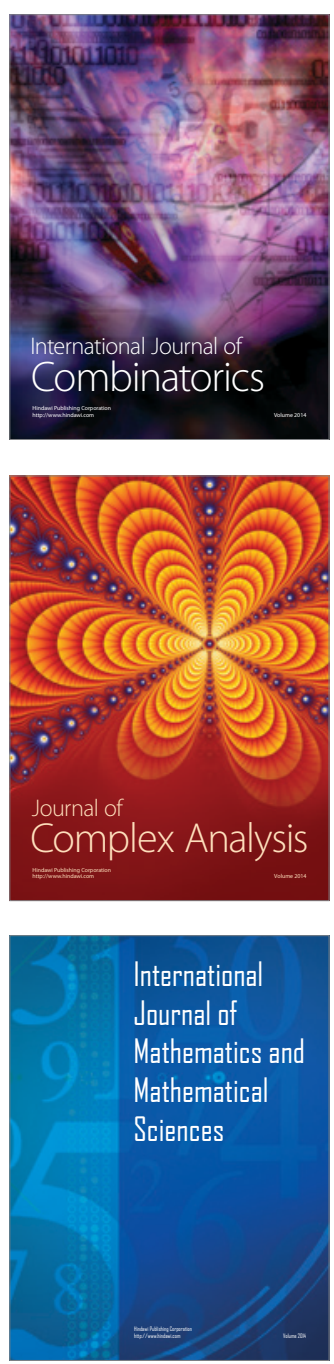
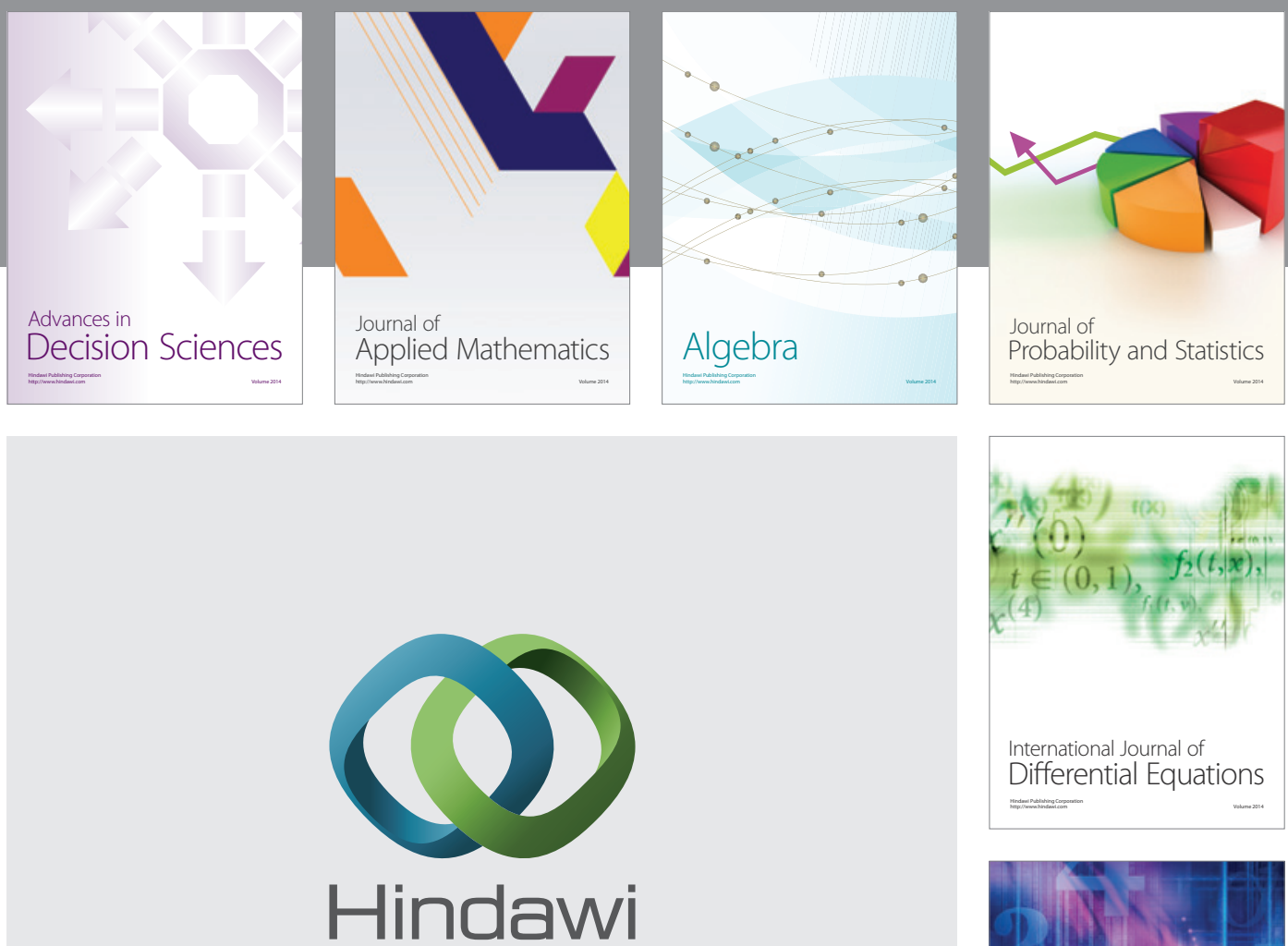

Submit your manuscripts at http://www.hindawi.com
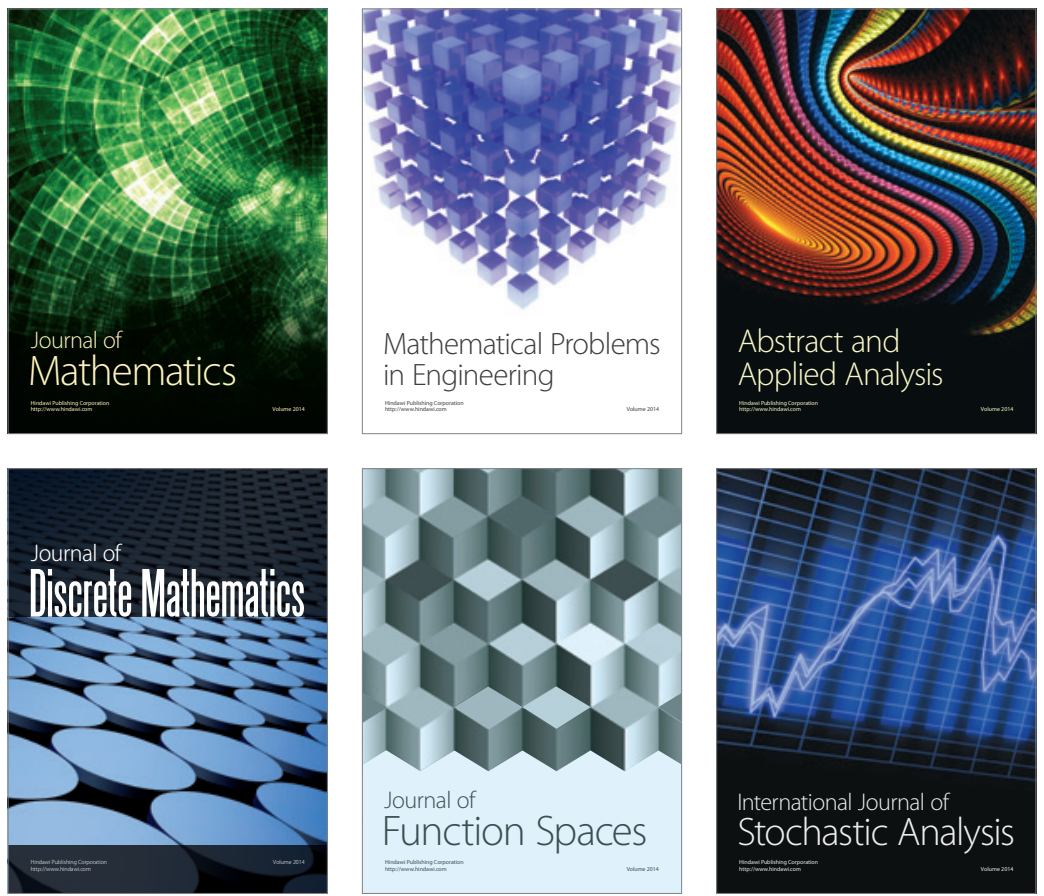

Journal of

Function Spaces

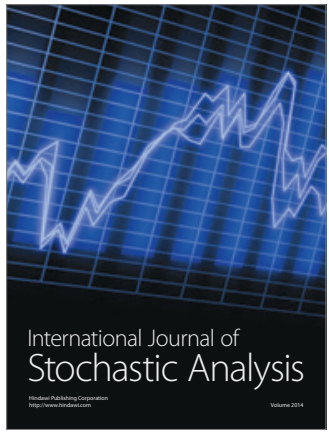

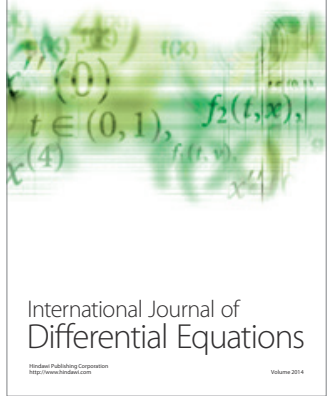
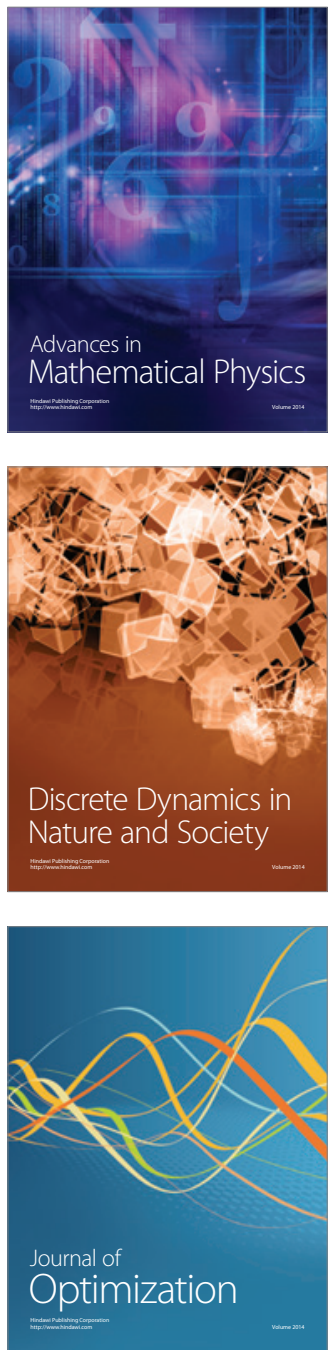